\title{
The Latest Developments in Imaging of Fibroblast Activation Protein
}

\author{
Annette Altmann ${ }^{1,2}$, Uwe Haberkorn ${ }^{1-3}$, and Jens Siveke ${ }^{4,5}$ \\ ${ }^{1}$ Department of Nuclear Medicine, University Hospital Heidelberg, Heidelberg, Germany; ${ }^{2}$ Clinical Cooperation Unit Nuclear \\ Medicine, German Cancer Research Center, Heidelberg, Germany; ${ }^{3}$ Translational Lung Research Center Heidelberg, German Center \\ for Lung Research, Heidelberg, Germany; ${ }^{4}$ Institute for Developmental Cancer Therapeutics, West German Cancer Center, University \\ Hospital Essen, Essen, Germany; and ${ }^{5}$ Division of Solid Tumor Translational Oncology, German Cancer Consortium, Essen, \\ Germany, and German Cancer Research Center, German Cancer Research Center, Heidelberg, Germany
}

Fibroblast activation protein (FAP), a membrane-anchored peptidase, is highly expressed in cancer-associated fibroblasts in more than $90 \%$ of epithelial tumors and contributes to progression and worse prognosis of different cancers. Therefore, FAP is considered a promising target for radionuclidebased approaches for diagnosis and treatment of tumors and for the diagnosis of nonmalignant diseases associated with a remodeling of the extracellular matrix. Accordingly, a variety of quinolone-based FAP inhibitors (FAPIs) coupled to chelators were developed displaying specific binding to human and murine FAP with a rapid and almost complete internalization. Because of a high tumor uptake and a very low accumulation in normal tissues, as well as a rapid clearance from the circulation, a high contrast is obtained for FAPI PET/CT imaging even at $10 \mathrm{~min}$ after tracer administration. Moreover, FAPI PET/CT provides advantages over ${ }^{18} \mathrm{~F}-\mathrm{FDG}$ PET/CT in several tumor entities for initial staging and detection of tumor recurrence and metastases, including peritonitis carcinomatosa.

Key Words: fibroblast-activating protein; PET; SPECT; imaging; theranostics; FAPI

J Nucl Med 2021; 62:160-167

DOI: 10.2967/jnumed.120.244806

$\mathbf{M}$

alignant tumors consist of neoplastic cells but also of a variety of nonmalignant cells that create and shape the tumor microenvironment, also called tumor stroma. These include cells of the basement membrane and capillaries, immune cells, and the heterogeneous population of fibroblastlike cells, termed cancer-associated fibroblasts (CAFs) (Fig. 1). Dysregulated interactions and signaling between tumor cells and cellular components of the tumor microenvironment - representing up to $90 \%$ of tumor tissue in frequently occurring carcinomas of the breast, colon, and pancreas (1)-

Received Sep. 2, 2020; revision accepted Oct. 21, 2020.

For correspondence or reprints contact: Uwe Haberkorn, Department of Nuclear Medicine, University Hospital Heidelberg, Im Neuenheimer Feld 400, 69120 Heidelberg, Germany.

E-mail: uwe.haberkorn@med.uni-heidelberg.de

Published online Oct. 30, 2020.

COPYRIGHT (c) 2021 by the Society of Nuclear Medicine and Molecular Imaging. significantly determine the malignant phenotype of cancer cells and contribute to tumor evolution and progression. Notably, through the production of various growth factors, chemokines, and cytokines, noncancerous stromal cells promote extracellular matrix remodeling, induction of angiogenesis, cellular migration, drug resistance, and evasion of immunosurveillance, facilitating invasion and the development of metastasis (2)

\section{CAFs}

CAFs, which account for most of the tumor stroma, have prominent desmoplastic reactions in colorectal cancer (3) and pancreatic cancer (4) and represent the most prominent modifiers of cancer progression and metastasis. Because of secretion of growth stimulatory, prosurvival, and angiogenic factors, they promote many different aspects of tumor development, including metabolic cooperation, remodeling of the extracellular matrix, induction of epithelial-to-mesenchymal transition, and therapy resistance. Moreover, the interaction of CAFs with cellular components of the immune system mediates immunosuppression and inflammation (5). CAFs are genetically more stable and, therefore, less susceptible to the development of therapy resistance than cancer cells per se.

CAFs originate from local fibroblasts, circulating fibroblasts, vascular endothelial cells via endothelial-to-mesenchymal transition, adipocytes, bone marrow-derived stem cells, or even cancer cells via endothelial-to-mesenchymal transition $(5,6)$. This spectrum of origin at least partially explains the phenotypically different subsets of CAFs that promote heterogeneous properties in individual tumor regions (6).

Irrespective of origin, the development of CAFs in the tumor stroma is accompanied by morphologic and molecular changes leading to spindle-shaped form and the expression of particular surface markers such as the $\alpha$-smooth muscle actin, the platelet-derived growth factor $\beta$, and the fibroblast activation protein (FAP) (6). Whereas many markers are heterogeneously expressed in CAFs in various tumor entities, FAP was shown to be upregulated in the stroma of more than $90 \%$ of epithelial carcinomas (7). 


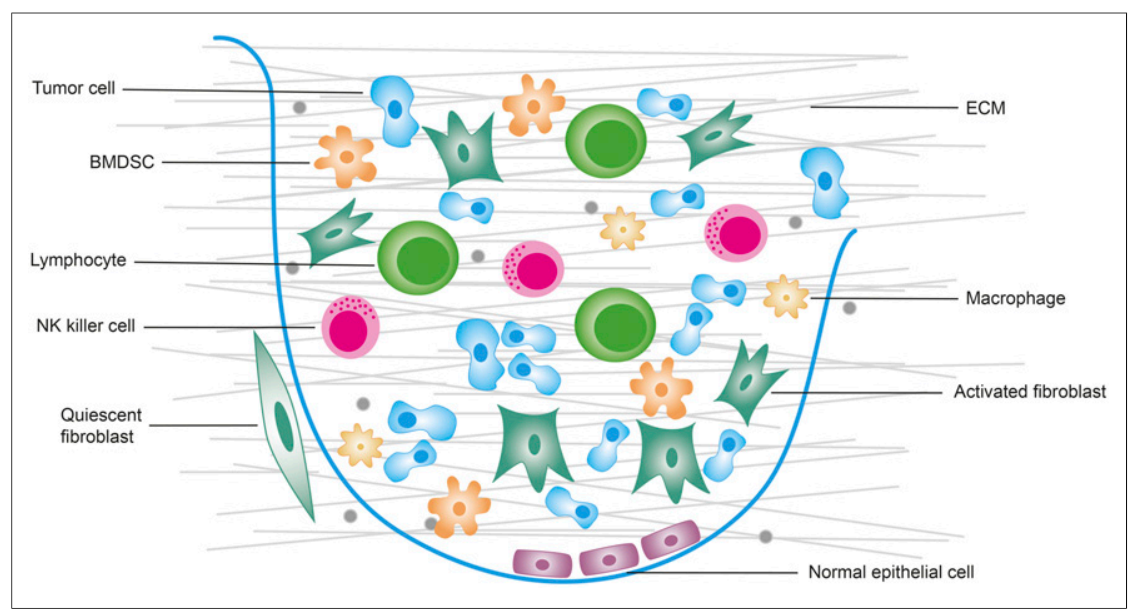

FIGURE 1. Tumor microenvironment consists of tumor cells and nontumor cells such as bone marrow-derived stem cells (BMDSC), B and T lymphocytes, natural killer (NK) cells, normal epithelial cells, activated fibroblasts (CAFs), and macrophages (T1 and T2). ECM = extracellular matrix. (Modified from (56).)

A recently published metaanalysis involving 15 studies that assessed FAP expression in 11 solid tumors by immunohistochemistry concluded that FAP was detected in 50\%$100 \%$ of the patients and that FAP expression is associated with increased local tumor invasion, increased risk of lymph node metastasis, and decreased survival (8). The association between FAP expression and poor prognosis was most clearly demonstrated in colorectal cancer and pancreatic cancer but was also shown in patients with hepatocellular cancer (9) and ovarian cancer (10). Therefore, FAP is considered an important target for diagnosis and therapeutic approaches.

\section{FAP}

FAP is a type II membrane-bound glycoprotein belonging to the dipeptidyl peptidase 4 family, with dipeptidyl peptidase and endopeptidase activity and sharing a 52\% identity at the protein level with dipeptidyl peptidase 4 (11). The protein, which was also given the name seprase, consists of 760 amino acids comprising a short intracellular domain (6 amino acids), a transmembrane domain (20 amino acids), and a large extracellular domain (734 amino acids). Both the postproline dipeptidyl peptidase and the endopeptidase activity of FAP depend on the catalytic triad consisting of serin (S624), aspartate (D702), and histidine $(\mathrm{H} 734)(12)$ and require homodimerization of the protein (13).

Further to overexpression in CAFs and certain transformed cells of malignant tissues, FAP is also selectively expressed in cells of benign diseases and in normal tissues during remodeling. These include stromal cells and mesenchymal stem cells during embryogenesis, wound healing, fibrotic reactions, and inflammatory conditions such as arthritis, atherosclerotic plaques, and fibrosis $(14,15)$, as well as in ischemic heart tissue after myocardial infarction
$(16,17)$. In healthy adult tissues, no or only insignificant levels of the protein are detected in the uterus, cervix, placenta, breast, and skin.

On the basis of enzymatic or nonenzymatic effects, FAP directly enhances proliferation, migration, and invasion of stromal fibroblasts and of additional cell types, including tumor, endothelial, and immune cells, leading to invasiveness, extracellular matrix degradation, tumor vascularization, and escape from immunosurveillance $(1,18)$. Accordingly, in many human malignancies, including colorectal cancer, pancreatic cancer, ovarian cancer, hepatocellular cancer, and non-smallcell lung carcinoma, high FAP expression is reported to correlate with increased local tumor invasion, increased risk of lymph node metastases, and decreased survival of patients (1).

\section{FAP-SPECIFIC TARGETING OF TUMORS}

The involvement of FAP in tumor development and progression, as well as the selective expression of the protein in the microenvironment of the most frequent tumors, led to the development of several FAP-based therapeutic approaches. Most of these strategies focused on the development of inhibitors of the enzymatic activity of the protein and, thereby, the contribution to growth and invasiveness of the tumor. The characterization of the dipeptide substrate requirements for FAP enabled the synthesis of a variety of different small molecules inhibiting the specific prolyl peptidase activity of the enzyme. Among these, talabostat, a boronic acid-based inhibitor (Val-proline boronic acid, PT100), the only FAP inhibitor (FAPI) tested in clinical studies so far, was well tolerated in solid tumor patients but, despite promising preclinical results, failed to induce tumor response in most malignancies in phase II clinical trials (19). A combinatorial treatment of talabostat with either docetaxel in non-small-cell lung carcinoma patients (20) or cisplatin in metastatic malignant melanoma patients (21) also failed to show any clinical benefit and even reduced the survival rate of these patients. Nevertheless, a variety of FAP-specific small molecules has recently been developed, providing the basis for new radiopharmaceuticals $(22,23)$.

Because of the conflicting data regarding the contribution of FAP's enzymatic activity to tumor progression, several therapeutic strategies have focused on the restricted tumor expression of FAP and the elimination of FAP-expressing cells. The unique proline-specific proteolytic activity of FAP was used to design FAP-activated prodrugs comprising a peptide containing the FAP cleavage site and a cytotoxin 
that, after systemic administration, circulates throughout the body in an inactive form $(24,25)$. On specific binding to FAP and subsequent proteolytic cleavage of the peptide, the cytotoxin is able to cross the cell membrane and reach its intracellular target in FAP-expressing cells but also nearby FAP-negative cancer cells (bystander effect). Although most of these strategies showed promising results in different xenograft models regarding tumor regression-and toxic side effects were markedly suppressed in comparison with the original drug-clinical application was limited by the toxicity of unprocessed protoxin or partial deposition of the active drug in healthy organs (9).

Clinical studies demonstrated the ability of FAP-specific antibodies to accumulate in tumor tissues after intravenous application. The ${ }^{131}$ I-labeled FAP-specific murine antibody ${ }^{19} \mathrm{~F}$ - originally used to indentify FAP as a tumor-associated antigen-displayed specific accumulation in tumor tissues of colon carcinoma patients. Thereafter, several humanized versions of ${ }^{19} \mathrm{~F}$ antibody were prepared and preclinically evaluated for their diagnostic and therapeutic properties in mouse models. Among these, the ${ }^{131}$ I-labeled anti-FAP antibody sibrotuzumab was applied for the treatment of patients with metastasized FAPpositive carcinomas, including breast cancer, colorectal cancer, and non-small-cell lung carcinoma, and tumor-specific accumulation was observed (26). However, ${ }^{131} \mathrm{I}$-sibrotuzumab revealed slow elimination from the liver, the spleen, and other normal organs, as was consistent with a slow blood-pool clearance. Although an antitumor activity of nonlabeled FAP antibodies was soon excluded, their excellent tumor stroma-targeting properties were exploited for the design of conjugates with toxins or immunomodulatory cytokines for localized delivery (27). AntiFAP antibodies and anti-FAP single-chain variable fragments and antibody fragments, respectively, coupled to highly toxic substances significantly improved the antitumor activity of the substance alone and stimulated the antitumor immune response in mouse tumor models (9).

Immune-based therapies targeting FAP also include the application of FAP-derived DNA, protein, and dendritic cell vaccines to elicit $\mathrm{CD} 8+-$ and $\mathrm{CD} 4+-$ mediated immune response against FAP-expressing cells (9) and the development of FAP-specific genetically engineered chimeric antigen receptor T cells (28). On the basis of T-cell-mediated release of proinflammatory cytokines and FAP-specific cytotoxicity, both therapeutic strategies led to elimination of FAP-positive cells in vitro and in different mouse cancer models and induced delayed tumor growth and prolonged survival of the respective animals. Moreover, the combination of FAP vaccination and chemotherapy improved the antitumor response in murine CT26 colon carcinoma and D2F2 breast cancer models (29). Nevertheless, the limited therapeutic effect combined with occasionally severe side effects has prevented the clinical transfer of these strategies so far.

\section{SMALL-MOLECULE INHIBITORS FOR IMAGING AND THERAPY}

The slow clearance of FAP antibodies leading to a high background signal results in a limited sensitivity for the detection of small lesions. This limited sensitivity can be countered by applying radiolabeled small molecules such as MIP-1232 or other FAPIs $(22,23,30)$. Therefore, a variety of quinoline-based (22) inhibitors were coupled to chelators and showed specific binding to human and murine FAP, with rapid and almost complete internalization (Fig. 2) (31-33). Because of high tumor uptake, a very low accumulation in normal tissues, and rapid clearance from the circulation, a high contrast is obtained for PET imaging even at 10 min after tracer administration (Fig. 3) (34,35). Usually, radioactivity was seen only in the renal pelvis and the bladder, with no accumulation in the renal parenchyma — characteristics that are favorable for a possible therapeutic application. Dose estimates for ${ }^{68} \mathrm{Ga}$-FAPI02, ${ }^{68} \mathrm{Ga}-\mathrm{FAPI}-04$, and ${ }^{68} \mathrm{Ga}-\mathrm{FAPI}-46$ ranged from 1.4 to 1.8 $\mathrm{mSv} / 100 \mathrm{MBq}(34,36)$ and, therefore, were comparable to other ${ }^{68} \mathrm{Ga}$-based tracers such as ${ }^{68} \mathrm{Ga}$-DOTATOC/DOTATATE or ${ }^{68} \mathrm{Ga}$-PSMA-11. A first comparison with ${ }^{18} \mathrm{~F}-\mathrm{FDG}$ in 6 patients with different tumor entities showed that, except for thyroid carcinoma, ${ }^{68} \mathrm{Ga}$-FAPI PET/CT showed a better contrast and a higher tumor uptake than ${ }^{18} \mathrm{~F}-\mathrm{FDG}$. This finding may be especially important in patients with peritonitis carcinomatosa (Fig. 4) (34).

Recently, several new ligands were developed. Moon et al. (37) modified a high-affinity inhibitor with squaric acid containing bifunctional DATA ${ }^{5 \mathrm{~m}}$ and DOTA chelators, which

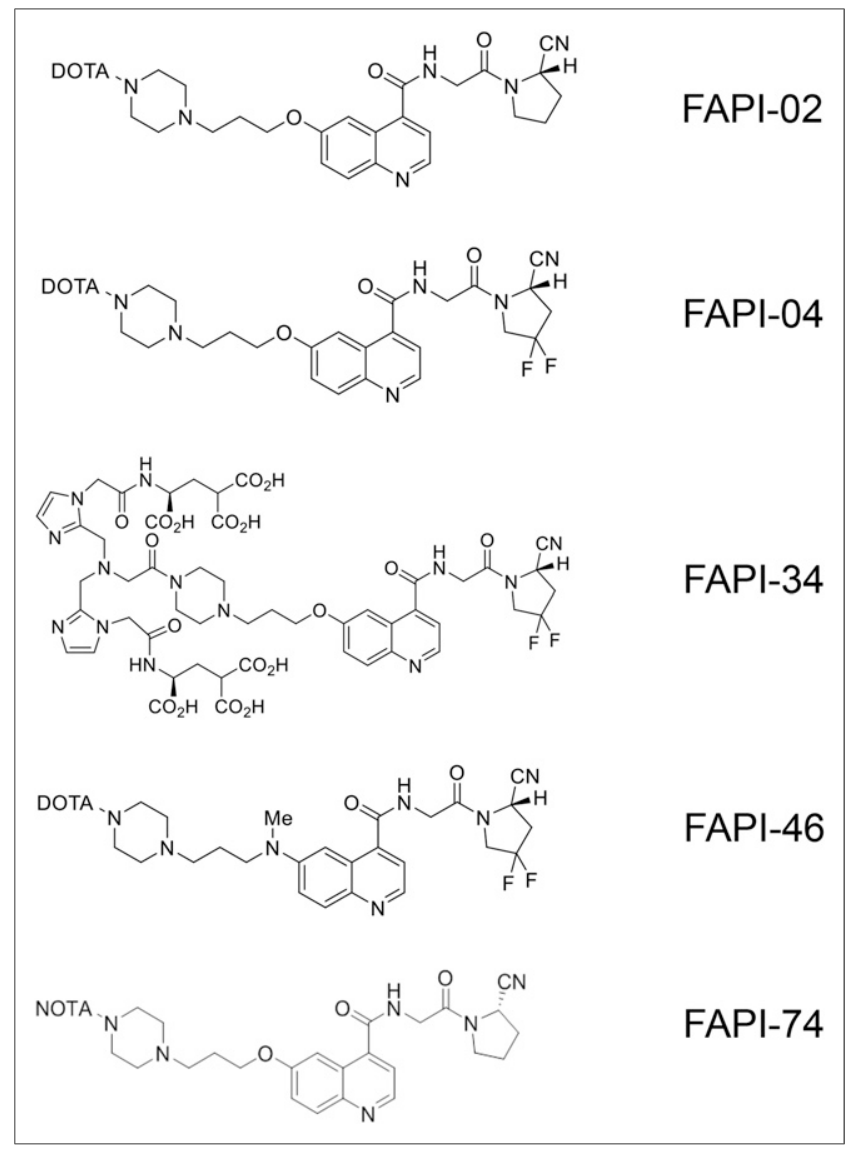

FIGURE 2. Structures of FAPI tracers used in clinical application. 


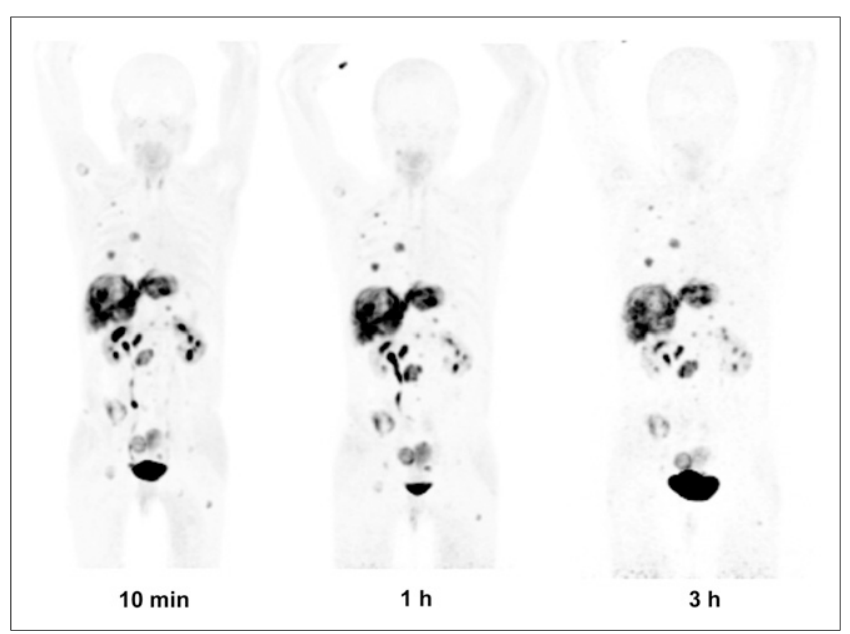

FIGURE 3. Maximum-intensity projections at $10 \mathrm{~min}, 1 \mathrm{~h}$, and $3 \mathrm{~h}$ after administration of FAPI-46 in patient with metastasized colorectal cancer. Because of rapid tumor uptake and very fast clearance, high-contrast images are possible even at 10 min after injection.

showed high tumor uptake and tissue contrast. Besides, ligands enabling large-scale production for clinical routine application were designed for ${ }^{18} \mathrm{~F}$ labeling $(38,39)$. Toms et al. (38) used an ${ }^{18} \mathrm{~F}$-labeled glycosylated FAPI $\left({ }^{18} \mathrm{~F}-\mathrm{FGlc}-\right.$ FAPI). Compared with ${ }^{68} \mathrm{Ga}$-FAPI-04 (half-maximal inhibitory concentration, $32 \mathrm{nM}$ ), the glycoconjugate, FGlc-FAPI (half-maximal inhibitory concentration, $167 \mathrm{nM}$ ), revealed

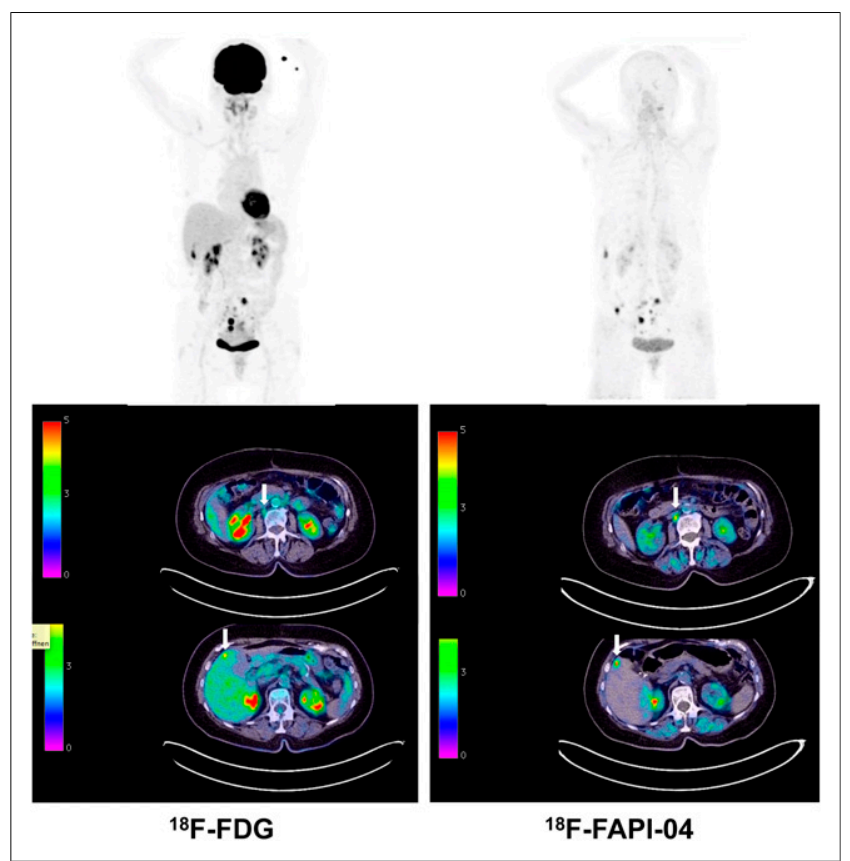

FIGURE 4. Comparison of ${ }^{18} \mathrm{~F}-\mathrm{FDG}$ PET/CT and FAPI-46 $\mathrm{PET} / \mathrm{CT}$ in patient with metastasized ovarian cancer. Shown are maximum-intensity projections and transaxial slices at $1 \mathrm{~h}$ after injection. Metastatic lesions in liver and lymph nodes are better seen with FAPI (arrows). lower affinity in vitro, higher plasma protein binding, and significant hepatobiliary excretion but higher tumor retention in the U87MG glioma model. Furthermore, ${ }^{18} \mathrm{~F}-$ FGlc-FAPI demonstrated high specific uptake in bones and joints. Giesel et al. (39) reported the use of the NOTAcoupled FAPI-74, which can be labeled with both ${ }^{18} \mathrm{~F}-\mathrm{AlF}$ and ${ }^{68} \mathrm{Ga}$, in 10 patients with lung cancer. A high contrast with an $\mathrm{SUV}_{\text {max }}$ of more than 10 was seen at $1 \mathrm{~h}$ after injection. Dosimetry in these patients revealed effective doses of $1.4 \pm$ $0.2 \mathrm{mSv} / 100 \mathrm{MBq}$ for ${ }^{18} \mathrm{~F}-\mathrm{FAPI}-74$ and $1.6 \mathrm{mSv} / 100 \mathrm{MBq}$ for ${ }^{68} \mathrm{Ga}$-FAPI-74 - doses that are lower than those obtained with ${ }^{18} \mathrm{~F}-\mathrm{FDG}$ and other ${ }^{18} \mathrm{~F}$ tracers.

Furthermore, new FAPI derivatives were designed for labeling with ${ }^{99 \mathrm{~m}} \mathrm{Tc}$ and ${ }^{188} \mathrm{Re}$, which are available from generators and can be used as a couple for diagnosis and FAPtargeted endoradiotherapy (40). The resulting ${ }^{99 \mathrm{~m} T \mathrm{Tc}-l a b e l e d}$ FAPI tracers showed excellent affinity (half-maximal inhibitory concentration, $6.4-12.7 \mathrm{nM}$ ) and binding properties in vitro and significant tumor uptake $(\leq 5.4$ percentage injected dose per gram) in biodistribution studies. The lead candidate, 99mTc-FAPI-34, was used for scintigraphy and SPECT in one patient with metastasized ovarian cancer and another patient with pancreatic cancer for follow-up of ${ }^{90}$ Y-FAPI-46 therapy (40). A comparison to PET/CT imaging with ${ }^{68} \mathrm{Ga}$-FAPI-46 revealed that ${ }^{99 \mathrm{~m} T c-F A P I-34}$ accumulated in the same tumor lesions, with excellent image quality.

In 80 patients who had 28 different tumor entities, with 54 primary tumors and 229 metastases, Kratochwil et al. (35) evaluated ${ }^{68} \mathrm{Ga}$-FAPI-02 and ${ }^{68} \mathrm{Ga}$-FAPI-04 uptake and discriminated 3 different groups: the highest average $\mathrm{SUV}_{\max }$ $(>12)$ was found in sarcoma, esophageal cancer, breast cancer, cholangiocarcinoma, and lung cancer; an intermediate $\mathrm{SUV}_{\max }$ (SUV, 6-12) was seen in hepatocellular, colorectal, head and neck, ovarian, pancreatic, and prostate cancer; and the lowest uptake (average $\mathrm{SUV}_{\max }<6$ ) was observed in pheochromocytoma, renal cell cancer, differentiated thyroid cancer, adenoid cystic cancer, and gastric cancer. Nevertheless, high variations in uptake were observed across and within all tumor entities.

In 75 patients with 12 different tumor entities, Chen et al. (41) compared ${ }^{68} \mathrm{Ga}$-DOTA-FAPI-04 PET/CT with ${ }^{18} \mathrm{~F}-\mathrm{FDG}$ PET/CT with respect to their performance in initial staging (51 patients) and in detecting tumor recurrence (21 patients). Because of its higher contrast, ${ }^{68} \mathrm{Ga}-\mathrm{FAPI}-04 \mathrm{PET} / \mathrm{CT}$ showed a higher detection rate in all 12 types of malignant tumors than did ${ }^{18}$ F-FDG PET/CT (98.2\% vs. $\left.82.1 \%, P=0.021\right)$. The sensitivity of ${ }^{68}$ Ga-FAPI PET/CT was also higher in lymph node metastases $(86.4 \%$ vs. $45.5 \%, P=0.004)$ and in bone and visceral metastases such as liver metastases, peritoneal carcinomatosis, and brain lesions $(83.8 \%$ vs. $59.5 \%, P=0.004)$.

The same group evaluated the value of ${ }^{68} \mathrm{Ga}$-FAPI PET/ $\mathrm{CT}$ in 68 patients with inconclusive ${ }^{18} \mathrm{~F}-\mathrm{FDG}$ PET/CT results for the discrimination of mass lesions seen on morphologic imaging $(n=18)$, for the detection of unknown primary $(n=6)$, for staging $(n=21)$, and for suspected 
recurrence $(n=23)$. Imaging was followed by histologic analysis as the gold standard. Most tumor lesions had higher uptake for ${ }^{68} \mathrm{Ga}$-FAPI than for ${ }^{18} \mathrm{~F}$-FDG and higher image contrast, leading to better tumor detection in suspected mass lesions and unknown primaries and better tumor staging and detection of disease recurrence, with an accuracy of $66.7 \%$, $66.7 \%, 33.3 \%$, and $87.0 \%$, respectively (42).

Furthermore, Shi et al. assessed the value of ${ }^{68} \mathrm{Ga}-$ FAPI-04 PET/CT in 17 patients with suspected hepatic nodules before surgery or biopsy and found high uptake in all tumor nodules, as opposed to only one benign nodule (43).

In patients with brain tumors (5 mutant isocitrate dehydrogenase [IDH] gliomas and 13 wild-type IDH glioblastomas), wild-type IDH glioblastomas and grade III or IV gliomas, but not grade II mutant IDH gliomas, showed elevated tracer uptake. In glioblastomas, spots were found with increased uptake in projection on MRI contrast-enhancing areas. Immunohistochemistry revealed FAP-positive cells with mainly elongated cell bodies and perivascular FAPpositive cells in glioblastomas and an anaplastic mutant IDH astrocytoma. If these data are confirmed in larger patient numbers, it seems possible that ${ }^{68} \mathrm{Ga}-\mathrm{FAPI}$ PET/CT may allow noninvasive distinction between low-grade mutant IDH and high-grade gliomas (44).

${ }^{68} \mathrm{Ga}-\mathrm{FAPI}$ PET/CT has also been used for radiation therapy planning in patients with glioblastoma, head and neck tumors, and tumors of the lower gastrointestinal tract (45-47). In 13 patients with glioblastoma, the target volume delineation with MRI and ${ }^{68} \mathrm{Ga}-\mathrm{FAPI}$ PET/CT showed significant variations resulting in a larger volume when ${ }^{68}$ Ga-FAPI PET/CT and MRI were used in combination for treatment planning (45).

Since head and neck cancers show a diffuse growth pattern, differentiation between tumor and healthy tissue can be challenging. Therefore, it can be expected that highcontrast images such as those obtained by ${ }^{68} \mathrm{Ga}$-FAPI PET/ CT may improve therapy planning. Syed et al. (46) used 4 different thresholds (3-, 5-, 7- and 10-fold increases in FAPI tumor-to-normal tissue ratio) to calculate 4 different gross tumor volumes, which were compared with gross tumor volumes obtained by contrast-enhanced CT and MRI. The quantitative analysis revealed high uptake in tumors (primarytumor $\mathrm{SUV}_{\max }, 14.62 \pm 4.44$ ) versus low uptake in healthy tissues such as the salivary glands $\left(\mathrm{SUV}_{\max }, 1.76 \pm 0.31\right)$. Concerning radiation planning, the gross tumor volume obtained with contrast-enhanced CT and MRI was $27.3 \mathrm{~cm}^{3}$, whereas FAPI-derived contours resulted in significantly different gross tumor volumes of $67.7,22.1,7.6$, and $2.3 \mathrm{~cm}^{3}$ for, respectively, 3-, 5-, 7- and 10-fold increases in FAPI. The combination of the morphologic methods with ${ }^{68} \mathrm{Ga}$-FAPI PET/CT revealed median volumes that were significantly larger with a 3-fold increase in FAPI $\left(54.7 \mathrm{~cm}^{3}, 200.5 \%\right.$ relative increase, $P=0.0005)$ and a 5-fold increase $(15.0$ $\mathrm{cm}^{3}, 54.9 \%$ increase, $P=0.0122$ ).

For tumors of the lower gastrointestinal tract $(n=22)$, a high tumor-to-background ratio of more than 3 was found in most cases. The TNM classification was changed in $50 \%$ of treatment-naïve patients $(n=6)$, and new lesions were seen in $47 \%$ of patients with metastases $(n=15)$. High, medium, and low changes in therapy were observed in $19 \%, 33 \%$, and $29 \%$ of patients, respectively. Furthermore, in almost all patients, delineation of the target volume improved when the image information from ${ }^{68} \mathrm{Ga}$-FAPI PET/ CT was used (47).

For all 3 tumor entities, future prospective studies are needed to assess the impact of ${ }^{68} \mathrm{Ga}$-FAPI PET/CT-based radiation therapy on tumor recurrence and survival. If these prospective studies turn out to be positive, the higher contrast between tumor and normal tissues may allow an automatically performed delineation of tumor volume and may thereby improve interphysician variability during therapy planning.

Besides its expression in malignant tumors, FAP expression is upregulated in a variety of benign diseases such as myocardial infarction, fibrosis of several organs, rheumatoid arthritis, and atherosclerotic plaques (14-17); an example of a patient with tumor and fibrosis is shown in Figure 5. For rheumatoid arthritis, murine models imaged with PET and SPECT using radiolabeled anti-FAP antibodies demonstrated high tracer uptake in the inflamed joints and a correlation between tracer accumulation and the severity of the disease (48-50).

In general, autoimmunity-triggered diseases are associated not only with inflammatory reactions but also with fibroblast activation, finally resulting in fibrosis and organ damage. In that respect, IgG4-related disease (IgG4-RD) is characterized by autoimmune inflammation associated with fibrosis predominantly in the pancreas, biliary tree, salivary glands, kidney, aorta, and other organs. Two studies evaluated the performance of ${ }^{68} \mathrm{Ga}-\mathrm{FAPI}$ PET/CT as compared with ${ }^{18} \mathrm{~F}$ FDG PET/CT in IgG4-RD $(51,52)$.

Using ${ }^{68} \mathrm{Ga}-F A P I$ PET/CT to examine 26 patients with 136 IgG4-RD-related lesions, Luo et al. (51) additionally detected disease-associated changes in $13 \%$ of the organs in $50 \%$ of these patients. This led to a higher detection rate of disease manifestation in pancreas, bile duct/liver, and lacrimal glands. In lesions, ${ }^{68} \mathrm{Ga}-\mathrm{FAPI}$ uptake was significantly higher than ${ }^{18} \mathrm{~F}$-FDG uptake, a finding that was attributed to the presence of activated fibroblasts. However, in IgG4related lymphadenopathy, FAPI showed no accumulation, a finding that was explained by a lack of fibrosis.

Using ${ }^{68}$ Ga-FAPI- and ${ }^{18}$ F-FDG PET/CT, as well as MRI and histopathologic assessment, Schmidkonz et al. (52) studied 27 patients with inflammatory, fibrotic, and overlapping manifestations of IgG4-related disease. Lesions that were positive on ${ }^{18} \mathrm{~F}-\mathrm{FDG}$ were found, on histology, to consist of dense infiltrations of $\mathrm{IgG} 4+$ cells, whereas FAPI-positive lesions consisted of abundant activated FAP-positive fibroblasts. Therefore, a discrimination between inflammatory and fibrotic activity seems possible. In addition, as compared with inflammatory lesions, fibrotic lesions showed a reduced response to antiinflammatory 

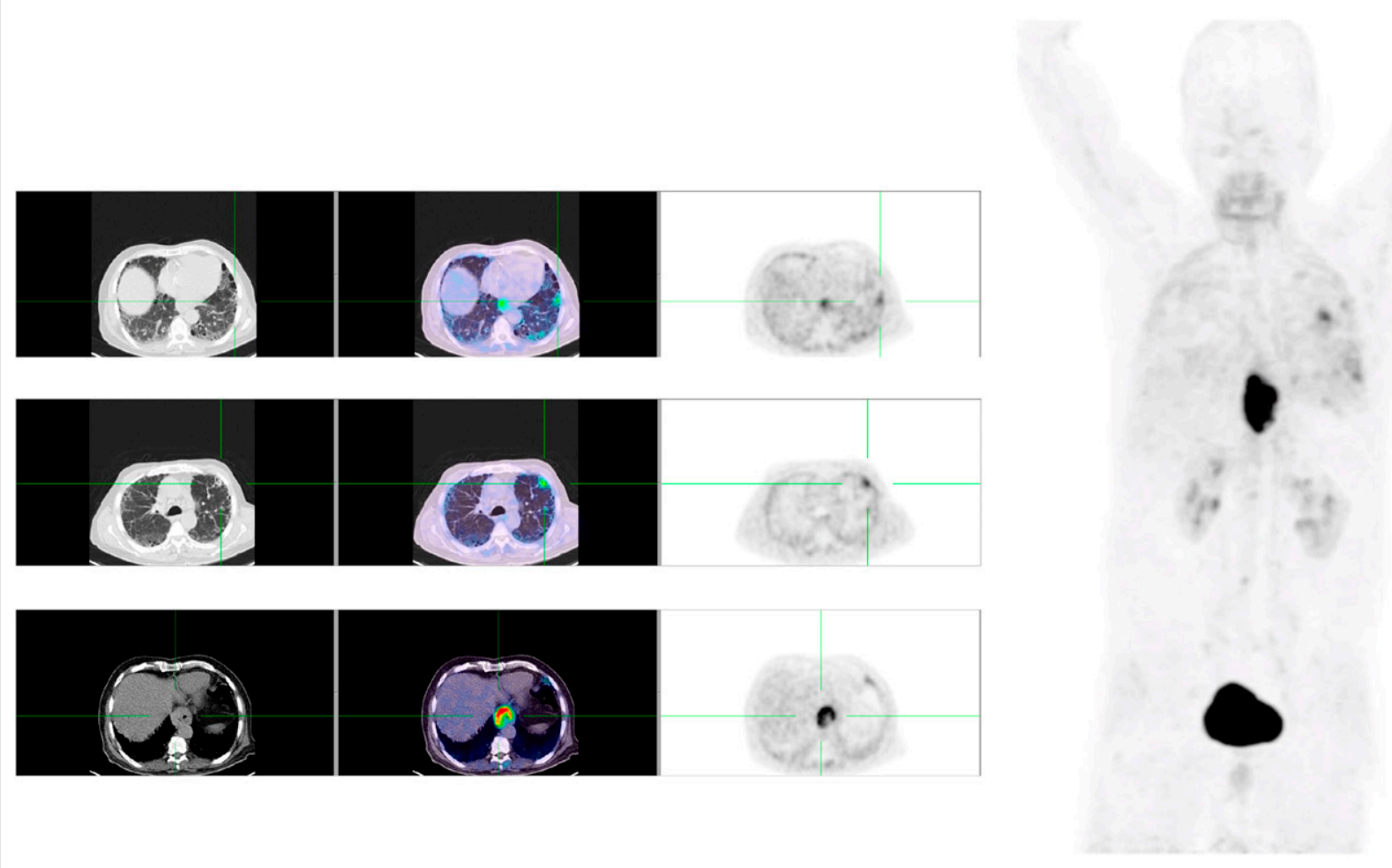

FIGURE 5. FAPI-04 PET/CT of patient with esophageal cancer and lung fibrosis. On left are transaxial slices (CT, fusion image, and PET alone, from left to right); on right is maximum-intensity projection.

treatment. The authors see potential for individualization of treatment strategies according to the extent of fibrosis or inflammation. If fibrosis is more extensive than inflammation, specific antifibrotic agents may be preferable to antiinflammatory therapy.

Another area of interest is cardiac disease. In an experimental setup, Varasteh et al. studied remodeling in rats after sham operation and coronary ligation to induce myocardial infarction (53). ${ }^{68} \mathrm{Ga}-\mathrm{FAPI}$ uptake in the diseased myocardium peaked at $6 \mathrm{~d}$ after coronary ligation, mainly localizing at the border of the infarction as shown by autoradiography and conventional staining. The FAPI-positive area was ${ }^{18} \mathrm{~F}-\mathrm{FDG}$-negative but positive for immunofluorescence in myofibroblasts. This finding may have a significant impact on diagnosis and the determination of prognosis, as well as on the clinical management of these patients.

A further possible application is mentioned in a case report of a patient with pancreatic cancer undergoing restaging after therapy with gemcitabine in combination with nab-paclitaxel followed by a modified protocol of folinic acid, fluorouracil, irinotecan, and oxaliplatin (54). Besides ${ }^{68} \mathrm{Ga}$-FAPI uptake in the primary tumor, in multiple liver metastases, and in peritoneal carcinomatosis, high tracer accumulation was seen in the left ventricular myocardium. The authors speculate that detection of myocardial damage after chemotherapy using ${ }^{68} \mathrm{Ga}$-FAPI PET/CT may be useful for identification and early management of cardiotoxicity.
Since the FAPI ligands are chelator-based and contain DOTA, these molecules may also be used for therapeutic applications. A first preclinical therapeutic application of ${ }^{225}$ Ac-FAPI-04 was performed on nude mice bearing human pancreatic PANC-1 cancer cells (55). In this model, high FAP expression was shown by small-animal PET imaging and immunohistochemistry. The therapy - an administration of $34 \mathrm{kBq}$ of ${ }^{225} \mathrm{Ac}-\mathrm{FAPI}-04$ - significantly delayed tumor growth, compared with nontreated controls, without significantly changing body weight. Clinically, few data exist for FAPI-04. In a first patient with metastasized final-stage breast cancer, FAPI-04 with ${ }^{90} \mathrm{Y}$ (half-life, $64 \mathrm{~h}$ ) was chosen to match the physical half-life of the therapeutic radionuclide to the tumor retention time. After a relatively low dose (2.9 GBq) of ${ }^{90}$ Y-FAPI-04, the metastases could be seen in Bremsstrahlung images even at $24 \mathrm{~h}$ after injection (33). Clinically, no therapy-related side effects and, especially, no hematotoxicity were observed, and a significant reduction in opioid dosing for pain was reported.

Because the origin, number, and distribution of FAPexpressing CAFs, as well as the number of FAP molecules per cell, may differ among tumors, we may expect variations in tumor uptake and intratumoral tracer distribution, potentially resulting in different pharmacokinetic profiles in different tumor entities. In a small series of patients with different tumor types, different kinetics from 1 to $3 \mathrm{~h}$ after injection was seen (32). If confirmed in a larger number of patients, this observation may have an impact on 
the indication for FAPI-based endoradiotherapy: tumors with a long retention of the radiopharmaceutical may respond better than tumors that eliminate it quickly. Furthermore, since differences in proteomes can be expected because of differences in the cellular origin of CAFs-with variations existing in the expression of different CAF markers, such as FAP, $\alpha$-smooth muscle actin, or plateletderived growth factor $\beta$-heterogeneity may be present in tracer uptake or in other FAP-targeting cells or molecules.

\section{CONCLUSION}

FAPIs are promising tracers for diagnostic applications in tumors showing a desmoplastic reaction, as well as for nonmalignant diseases associated with tissue remodeling, such as myocardial infarction; sarcoidosis; chronic inflammation; fibrosis of the lung, liver, and kidney; rheumatoid arthritis; and possibly atherosclerosis. For FAPI-based endoradiotherapy, the physical half-life of the radionuclide has to be adjusted to the retention time: radionuclides with shorter half-lives seem preferable to those with longer halflives. Thus, the use of ${ }^{188} \mathrm{Re},{ }^{153} \mathrm{Sm},{ }^{213} \mathrm{Bi}$, or ${ }^{212} \mathrm{~Pb}$ would be favored. Since CAFs are involved in many tumor-supporting processes, such as angiogenesis, chemoresistance, and resistance to immunotherapy, combinations of FAP-targeted endoradiotherapy with radiation therapy, chemotherapy, and immunotherapy may have a synergizing effect and represent an exciting future research area.

\section{DISCLOSURE}

There is a patent application (EP 18155420.5) for quinoline-based FAP-targeting agents for imaging and therapy in nuclear medicine (Uwe Haberkorn). Jens Siveke receives research funding from Celgene and BMS; receives personal fees from AstraZeneca, BMS, Celgene, Immunocore, Novartis, and Roche; and holds ownership in FAPI Holding $(<3 \%)$. Uwe Haberkorn holds ownership in FAPI Holding $(<3 \%)$. No other potential conflict of interest relevant to this article was reported.

\section{REFERENCES}

1. Zi F, He J, He D, et al. Fibroblast activation protein $\alpha$ in tumor microenvironment: recent progression and implications. Mol Med Rep. 2015;11:3203-3211.

2. Bussard KM, Mutkus L, Stumpf K, et al. Tumor-associated stromal cells as key contributors to the tumor microenvironment. Breast Cancer Res. 2016;18:84

3. Henry LR, Lee HO, Lee JS, et al. Clinical implications of fibroblast activation protein in patients with colon cancer. Clin Cancer Res. 2007;13:1736-1741.

4. Cohen SJ, Alpaugh RK, Palazzo I, et al. Fibroblast activation protein and its relationship to clinical outcome in pancreatic adenocarcinoma. Pancreas. 2008;37:154-158.

5. Gascard P, Tlsty TD. Carcinoma-associated fibroblasts: orchestrating the composition of malignancy. Genes Dev. 2016;30:1002-1019.

6. Bu L, Baba H, Yoshida N, et al. Biological heterogeneity and versatility of cancer-associated fibroblasts in the tumor microenvironment. Oncogene. 2019;38: 4887-4901.

7. Scanlan MJ, Raj BK, Calvo B, et al. Molecular cloning of fibroblast activation protein alpha, a member of the serine protease family selectively expressed in stromal fibroblasts of epithelial cancers. Proc Natl Acad Sci USA. 1994;91:5657-5661.

8. Liu F, Qi L, Liu B, et al. Fibroblast activation protein overexpression and clinical implications in solid tumors: a meta-analysis. PLoS One. 2015;10:e0116683.
9. Busek P, Mateu R, Zubal M, et al. Targeting fibroblast activation protein in cancer: prospects and caveats. Front Biosci (Landmark Ed). 2018;23:1933-1968.

10. Puré E, Blomberg R. Pro-tumorigenic roles of fibroblast activation protein in cancer: back to the basics. Oncogene. 2018;37:4343-4357.

11. Hamson EJ, Keane FM, Tholen S, et al. Understanding fibroblast activation protein (FAP): substrates, activities, expression and targeting for cancer therapy. Proteomics Clin Appl. 2014;8:454-463.

12. Goldstein LA, Ghersi G, Pineiro-Sanchez ML, et al. Molecular cloning of seprase: a serine integral membrane protease from human melanoma. Biochim Biophys Acta. 1997;1361:11-19.

13. Ghersi G, Zhao Q, Salamone M, et al. The protease complex consisting of dipeptidyl peptidase IV and seprase plays a role in the migration and invasion of human endothelial cells in collagenous matrices. Cancer Res. 2006;66: 4652-4661.

14. Egger C, Cannet C, Gerard C, et al. Effects of the fibroblast activation protein inhibitor, PT100, in a murine model of pulmonary fibrosis. Eur J Pharmacol. 2017;809:64-72.

15. Uitte de Willige S, Malfliet JJ, Janssen HL, et al. Increased N-terminal cleavage of alpha-2-antiplasmin in patients with liver cirrhosis. J Thromb Haemost. 2013;11:2029-2036.

16. Tillmanns J, Hoffmann D, Habbaba Y, et al. Fibroblast activation protein alpha expression identifies activated fibroblasts after myocardial infarction. $\mathrm{J} \mathrm{Mol} \mathrm{Cell}$ Cardiol. 2015;87:194-203.

17. Nagaraju CK, Dries E, Popovic N, et al. Global fibroblast activation throughout the left ventricle but localized fibrosis after myocardial infarction. Sci Rep. 2017;7:10801.

18. Jacob M, Chang L, Pure E. Fibroblast activation protein in remodeling tissues. Curr Mol Med. 2012;12:1220-1243.

19. Narra K, Mullins SR, Lee HO, et al. Phase II trial of single agent ValboroPro (Talabostat) inhibiting fibroblast activation protein in patients with metastatic colorectal cancer. Cancer Biol Ther. 2007;6:1691-1699.

20. Eager RM, Cunningham CC, Senzer NN, et al. Phase II trial of talabostat and docetaxel in advanced non-small cell lung cancer. Clin Oncol (R Coll Radiol). 2009;21:464-472.

21. Eager RM, Cunningham CC, Senzer NN, et al. Phase II assessment of talabostat and cisplatin in second-line stage IV melanoma. BMC Cancer. 2009;9:263.

22. Jansen K, Heirbaut L, Cheng JD, et al. Selective inhibitors of fibroblast activation protein (FAP) with a (4-quinolinoyl)-glycyl-2-cyanopyrrolidine scaffold. ACS Med Chem Lett. 2013;4:491-496.

23. Jansen K, Heirbaut L, Verkerk R, et al. Extended structure-activity relationship and pharmacokinetic investigation of (4-quinolinoyl)glycyl-2-cyanopyrrolidine inhibitors of fibroblast activation protein (FAP). J Med Chem. 2014;57: 3053-3074.

24. Brennen WN, Isaacs JT, Denmeade SR. Rationale behind targeting fibroblast activation protein-expressing carcinoma-associated fibroblasts as a novel chemotherapeutic strategy. Mol Cancer Ther. 2012;11:257-266.

25. Ke M-R, Chen S-F, Peng X-H, et al. A tumor-targeted activatable phthalocyanine-tetrapeptide-doxorubicin conjugate for synergistic chemophotodynamic therapy. Eur J Med Chem. 2017;127:200-209.

26. Sounni N E, Noel A. Targeting the tumor microenvironment for cancer therapy. Clin Chem. 2013;59:85-93.

27. Ostermann E, Garin-Chesa P, Heider KH, et al. Effective immunoconjugate therapy in cancer models targeting a serine protease of tumor fibroblasts. Clin Cancer Res. 2008;14:4584-4592.

28. Lo A, Wang L-CS, Scholler J, et al. Tumor-promoting desmoplasia is disrupted by depleting FAP-expressing stromal cells. Cancer Res. 2015;75:2800-2810.

29. Loeffler M, Krüger JA, Niethammer AG, et al. Targeting tumor-associated fibroblasts improves cancer chemotherapy by increasing intratumoral drug uptake. J Clin Invest. 2006;116:1955-1962.

30. Zimmermann C, Babich JW, Joyal J, Marquis J, Wang J-C, inventors; Molecular Insight Pharmaceuticals, Inc., assignee. Selective seprase inhibitors. US patent 2010/0098633 A1. April 22, 2010.

31. Loktev A, Lindner T, Mier W, et al. A new method for tumor imaging by targeting cancer associated fibroblasts. J Nucl Med. 2018;59:1423-1429.

32. Loktev A, Lindner T, Burger EM, et al. Development of novel FAP-targeted radiotracers with improved tumor retention. J Nucl Med. 2019;60:1421-1429.

33. Lindner T, Loktev A, Altmann A, et al. Development of quinoline based theranostic ligands for the targeting of fibroblast activation protein. $J$ Nucl Med. 2018;59:1415-1422.

34. Giesel FL, Kratochwil C, Lindner T, et al. FAPI-PET/CT: biodistribution and preliminary dosimetry estimate of two DOTA-containing FAP-targeting agents in patients with various cancers. J Nucl Med. 2019;60:386-392. 
35. Kratochwil C, Flechsig P, Lindner T, et al. FAPI-PET/CT: mean intensity of tracer-uptake (SUV) in 28 different kinds of cancer. J Nucl Med. 2019;60: 801-805.

36. Meyer C, Dahlbom M, Lindner T, et al. Radiation dosimetry and biodistribution of ${ }^{68} \mathrm{Ga}$-FAPI-46 PET imaging in cancer patients. J Nucl Med. 2020;61: 1171-1177.

37. Moon ES, Elvas F, Vliegen G, et al. Targeting fibroblast activation protein (FAP): next generation PET radiotracers using squaramide coupled bifunctional DOTA and DATA $(5 \mathrm{~m})$ chelators. EJNMMI Radiopharm Chem. 2020;5:19.

38. Toms J, Kogler J, Maschauer S, et al. Targeting fibroblast activation protein: radiosynthesis and preclinical evaluation of an ${ }^{18} \mathrm{~F}$-labeled FAP inhibitor. $\mathrm{J}$ Nucl Med. April 24, 2020 [Epub ahead of print].

39. Giesel F, Adeberg S, Syed M, et al. FAPI-74 PET/CT using either ${ }^{18}$ F-AlF or coldkit ${ }^{68} \mathrm{Ga}$-labeling: biodistribution, radiation dosimetry and tumor delineation in lung cancer patients. J Nucl Med. June 26, 2020 [Epub ahead of print].

40. Lindner T, Altmann A, Kraemer S, et al. Design and development of ${ }^{99 \mathrm{~m}} \mathrm{Tc}$ labeled FAPI-tracers for SPECT-imaging and ${ }^{188}$ Re therapy. J Nucl Med. March 13, 2020 [Epub ahead of print].

41. Chen H, Pang Y, Wu J, et al. Comparison of $\left({ }^{68} \mathrm{Ga}\right)$ Ga-DOTA-FAPI-04 and $\left({ }^{18} \mathrm{~F}\right)$ FDG PET/CT for the diagnosis of primary and metastatic lesions in patients with various types of cancer. Eur J Nucl Med Mol Imaging. 2020;47:1820-1832.

42. Chen H, Zhao L, Ruan D, et al. Usefulness of $\left[{ }^{68} \mathrm{Ga}\right] \mathrm{Ga}$-DOTA-FAPI-04 PET/CT in patients presenting with inconclusive $\left[{ }^{18} \mathrm{~F}\right] \mathrm{FDG}$ PET/CT findings. Eur J Nucl Med Mol Imaging. June 25, 2020 [Epub ahead of print].

43. Shi X, Xing H, Yang X, et al. Fibroblast imaging of hepatic carcinoma with ${ }^{68} \mathrm{Ga}$-FAPI-04 PET/CT: a pilot study in patients with suspected hepatic nodules. Eur J Nucl Med Mol Imaging. May 29, 2020 [Epub ahead of print].

44. Röhrich M, Loktev A, Wefers AK, et al. IDH-wildtype glioblastomas and grade III/IV IDH-mutant gliomas show elevated tracer uptake in fibroblast activation protein-specific PET/CT. Eur J Nucl Med Mol Imaging. 2019;46:2569-2580.

45. Windisch P, Röhrich M, Regnery S, et al. Fibroblast activation protein (FAP) specific PET for advanced target volume delineation in glioblastoma. Radiother Oncol. 2020;150:159-163.
46. Syed M, Flechsig P, Liermann J, et al. Fibroblast activation protein inhibitor (FAPI) PET for diagnostics and advanced targeted radiotherapy in head and neck cancers. Eur J Nucl Med Mol Imaging. 2020;47:2836-2845.

47. Koerber SA, Staudinger F, Kratochwil C, et al. The role of FAPI-PET/CT for patients with malignancies of the lower gastrointestinal tract: first clinical experience. J Nucl Med. February 14, 2020 [Epub ahead of print].

48. Laverman P, van der Geest T, Terry SY, et al. Immuno-PET and immunoSPECT of rheumatoid arthritis with radiolabeled anti-fibroblast activation protein antibody correlates with severity of arthritis. J Nucl Med. 2015;56: 778-783.

49. Terry SY, Koenders MI, Franssen GM, et al. Monitoring therapy response of experimental arthritis with radiolabeled tracers targeting fibroblasts, macrophages, or integrin $\alpha_{\mathrm{v}} \beta_{3}$. J Nucl Med. 2016;57:467-472.

50. van der Geest T, Laverman P, Gerrits D, et al. Liposomal treatment of experimental arthritis can be monitored noninvasively with a radiolabeled antifibroblast activation protein antibody. $J$ Nucl Med. 2017;58:151-155.

51. Luo Y, Pan Q, Yang H, et al. Fibroblast activation protein targeted PET/CT with ${ }^{68} \mathrm{Ga}$-FAPI for imaging IgG4-related disease: comparison to ${ }^{18} \mathrm{~F}$-FDG PET/CT. $J$ Nucl Med. June 8, 2020 [Epub ahead of print].

52. Schmidkonz C, Rauber S, Atzinger A, et al. Disentangling inflammatory from fibrotic disease activity by fibroblast activation protein imaging. Ann Rheum Dis. July 21, 2020 [Epub ahead of print].

53. Varasteh Z, Mohanta S, Robu S, et al. Molecular imaging of fibroblast activity after myocardial infarction using a ${ }^{68} \mathrm{Ga}$-labeled fibroblast activation protein inhibitor, FAPI-04. J Nucl Med. 2019;60:1743-1749.

54. Totzeck M, Siebermair J, Rassaf T, et al. Cardiac fibroblast activation detected by positron emission tomography/computed tomography as a possible sign of cardiotoxicity. Eur Heart J. 2020;41:1060.

55. Watabe T, Liu Y, Kaneda-Nakashima K, et al. Theranostics targeting fibroblast activation protein in the tumor stroma: ${ }^{64} \mathrm{Cu}$ - and ${ }^{225}$ Ac-labeled FAPI-04 in pancreatic cancer xenograft mouse models. J Nucl Med. 2020;61:563-569.

56. Kalluri R. The biology and function of fibroblasts in cancer. Nat Rev Cancer. 2016;16:582-598. 